\title{
Treatment of neurosyphilis with ceftriaxone
}

\author{
S Shann, J Wilson
}

Sex Transm Infect 2003;79:415-416

The first choice treatment for late syphilis is penicillin. Other than doxycycline, which penetrates the CNS, there are few alternatives for the treatment of neurosyphilis. We report a case of successful treatment of symptomatic neurosyphilis with parenteral ceftriaxone.

$\mathrm{T}$ he first choice treatment for late syphilis is penicillin. ${ }^{1}$ Other than doxycycline, which penetrates the CNS, there are few alternatives for the treatment of neurosyphilis.

We report a case of successful treatment of symptomatic neurosyphilis with parenteral ceftriaxone.

\section{CASE REPORT}

A 61 year old heterosexual man who had served in the armed forces abroad was referred to the department of genitourinary medicine with a diagnosis of symptomatic neurosyphilis in September 1997.

Six months before this he had been admitted to hospital with a history of expressive dysphasia and a left sided hemiparesis. His behaviour had been noted to be "out of character" for a week before the admission. A computed tomograph (CT) scan revealed patchy ischaemic change, especially of the left frontal lobe. A carotid duplex scan and ECG were normal but an echocardiogram revealed a trivial degree of aortic regurgitation. His neurological abnormalities resolved over several days and he was discharged.

In June 1997 he was reviewed in the neurology outpatient clinic with a history of transient dysphasia and impaired use of his right hand. He had developed marked short term memory loss and was unable to perform simple tasks. On examination he was generally tremulous and dysarthric but with normal tendon reflexes, tone, power, pain, and vibration sensation and proprioception. A magnetic resonance imaging (MRI) scan revealed generalised prominence of CSF spaces disproportionate to the patient's age and small vessel ischaemic damage within both frontal lobes.

He was readmitted to hospital in August 1997 with a history of frequent falls. He was confused and had delusions of persecution. Investigations revealed positive syphilis serology in blood and CSF (see table 1) and he was referred to the department of genitourinary medicine.

A diagnosis of untreated symptomatic neurosyphilis was made with both cerebrovascular components and parenchymatous features of general paresis. He had a history of anaphylaxis with penicillin and there were concerns about non-compliance with doxycycline. We decided to use ceftriaxone because of its good CNS penetration. There is a $10 \%$ risk of cross sensitivity between penicillin and cephalosporins; therefore, he was admitted to hospital and given a test dose of $50 \mathrm{mg}$ of ceftriaxone intravenously with full resuscitation facilities available. He was commenced on prednisolone $10 \mathrm{mg}$ three times daily for 24 hours before and 48 hours after starting treatment because of the risk of a Jarisch-Herxheimer reaction causing neurological deteriora- tion or acute coronary ostitis. He received $1 \mathrm{~g}$ of ceftriaxone once daily for 14 days.

He was reviewed 7, 19, and 36 months after treatment with repeat syphilis serology of blood and CSF (see table 1). Clinically he made some improvement in terms of his memory, speech, and mobility.

\section{DISCUSSION}

The recommended treatment for neurosyphilis is penicillin. Doxycycline and amoxycillin have been evaluated as second line options but as they are given orally, there may be adherence issues. The UK national guidelines ${ }^{2}$ and the European guidelines for the management of syphilis ${ }^{3}$ do not recommend ceftriaxone for the treatment of late syphilis. However, the CDC Sexually Transmitted Diseases Treatment Guidelines $2002^{4}$ suggest that it may be used as an alternative in penicillin allergic patients with neurosyphilis.

Ceftriaxone has been proved to have good CNS penetration. A dose of $1 \mathrm{~g}$ daily achieves levels well above the MIC for Treponema pallidum of $0.0006 \mu \mathrm{g} / \mathrm{ml} .^{5}$ It also differs from other cephalosporins by having an unusually long serum half life of approximately 7 hours. ${ }^{6}$

However there have been reports that intramuscular ceftriaxone may not be adequate treatment for neurosyphilis. ${ }^{5}$ A retrospective study of HIV infected individuals treated with ceftriaxone for asymptomatic neurosyphilis or latent syphilis revealed a $23 \%$ failure rate. ${ }^{7}$ Another study suggested that intravenous ceftriaxone may be an alternative to penicillin for treatment of HIV infected patients with neurosyphilis complicating early syphilis infection. However, in this setting, disease may be confined to the meninges and

Table 1 Blood and CSF parameters before and after treatment with ceftriaxone

\begin{tabular}{lllll}
\hline & Sept 97 & April 98 & April 99 & Sept 00 \\
\hline Blood & & & & \\
TPHA* & 1 in 256 & 1 in 128 & 1 in 64 & $>1$ in 256 \\
FTA abs & Pos & Pos & Pos & Pos \\
RPR & 1 in 128 & 1 in 32 & 1 in 64 & 1 in 16 \\
Albumin (g/l) & 41 & 40 & - & 44 \\
IgG (g/l) & 14.1 & 9.9 & - & 15 \\
Glucose (mmol/l) & 4.3 & 3.7 & - & - \\
CSF & & & & \\
TPHA* & 1 in 64 & 1 in 32 & 1 in 32 & $>1$ in 256 \\
FTA abs & Pos & Pos & Pos & Pos \\
RPR & 1 in 4 & neg & 1 in 2 & neg \\
Protein (g/l) & 2.03 & 0.63 & - & 0.55 \\
Albumin (mg/l) & - & 418 & - & 262 \\
IgG (mg/l) & - & 97 & $>120$ & 81 \\
White cells (/l) & $32 \times 10^{6}$ & $<1 \times 10^{6}$ & - & $<1 \times 10^{6}$ \\
Glucose (mmol/l) & 2.5 & 2.8 & - & - \\
IgG index $(<0.70)$ & - & 0.94 & - & 0.91 \\
Albumin quotient & - & 10.45 & - & 5.95 \\
(<7.8) & & & & \\
\hline
\end{tabular}

$\mathrm{TPHA}=$ Treponema pallidum haemagglutination; FTA = fluorescent treponemal antibody; $R P R=$ rapid plasmin reagin; TPPA = Treponema pallidum particle agglutination.

*In January 2000 the laboratory began monitoring TPPA instead of TPHA. 
may be easier to cure than disease of longer duration that could involve meninges and brain parenchyma. ${ }^{8}$

The patient in our case had evidence of both cerebrovascular and parenchymal disease. He received 14 days of ceftriaxone ( 3 days intravenously and 11 days intramuscularly) and was followed up for 36 months with blood and CSF analysis. His blood RPR fell from 1 in 128 pre-treatment to 1 in 16 (a threefold reduction) 36 months after treatment. His CSF TPHA and FTA abs have remained detectable but the RPR was negative on the last occasion. The protein level in the CSF has consistently declined from $2.03 \mathrm{~g} / \mathrm{l}$ to $0.55 \mathrm{~g} / \mathrm{l}$ as have the CSF albumin and IgG levels. The IgG index remains elevated but the albumin quotient has normalised. Even after successful treatment these latter two parameters may remain abnormal. $^{3}$

There are few other reports of successful treatment of symptomatic neurosyphilis in HIV negative individuals with ceftriaxone. There is a single case of treatment of asymptomatic neurosyphilis ${ }^{9}$ and of treatment of meningomyelitis complicating secondary syphilis. ${ }^{10}$ Our case suggests that ceftriaxone may be a useful alternative in HIV negative patients with neurosyphilis, but because of the doubt about its efficacy in those who are co-infected, a larger study with CSF levels of ceftriaxone should be performed.

\section{Authors' affiliations}

S Shann, J Wilson, Department of Genitourinary Medicine, Leeds General Infirmary, Great George Street, Leeds LSI 3EX, UK
Correspondence to: Dr S Shann, Department of Genitourinary Medicine, Sunnybank Wing, Leeds General Infirmary, Great George Street, Leeds LS1 3EX, UK; siobhan.mcmyler@leedsth.nhs.uk

Accepted for publication 27 February 2003

\section{REFERENCES}

1 Hahn RD, Webster B, Weickhardt G, et al. The results of treatment of 1086 general paralytics the majority of whom were followed up for more than 5 years. J Chron Dis 1958;7:209-27.

2 Clinical Effectiveness Group. UK national guidelines on STIs and closely related conditions. Sex Transm Infect 1999;75(Supp I).

3 Goh BT, Van Voorst Vader PC. European guideline for the management of syphilis. Int J STD AIDS 2001;12(suppl 3):14-26.

4 Centers For Disease Control And Prevention. Sexually transmitted diseases treatment guidelines 2002. MMWR 2002;51(RR-6):18-26.

5 Marra CM, Slatter V, Tartaglione TA, et al. Evaluation of aqueous penicillin $G$ and ceffriaxone for experimental neurosyphilis. (lefter) $J$ Infect Dis 1992;165:396-7.

6 Steele RW. Ceftriaxone therapy of meningitis and serious infections. Am J Med 1984;77(4C): $50-3$.

7 Dowell ME, Ross PG, Musher DM, et al. Response of latent syphilis or neurosyphilis to ceftriaxone therapy in persons infected with human immunodeficiency virus. Am J Med 1992;93:481-8.

8 Marra CM, Boutin P, McArthur JC, et al. A pilot study evaluating ceftriaxone and penicillin $\mathrm{G}$ as treatment agents for neurosyphilis in human immunodeficiency virus-infected individuals. Clin Infect Dis 2000;30:540-4.

9 Hook EW, Baker-Zander SA, Moskovitz BL, et al. Ceftriaxone therapy for asymptomatic neurosyphilis. Case report and Western blot analysis of serum and cerebrospinal fluid lgG response to therapy. Sex Transm Dis 1986;13(Suppl 3):185-8.

10 Gentile JH, Viviani C, Sparo MD, et al. Syphilitic meningomyelitis treated with ceftriaxone: case report. Clin Infect Dis 1998;26:528. 